\title{
准噶尔盆地植被与土壤盐渍化关联性变化趋势分析
}

\author{
杜秉晨曦，程勇翔*，吴玲 \\ 石河子大学,生命科学学院, 石河子 832000
}

\begin{abstract}
摘要: 准噶尔盆地作为北疆地区主要的气候单元其环境变化会影响北疆地区整体的的生态环境变化。植被作为衡量区域生态 环境的重要指标直接反映了准噶尔盆地的生态状况,近年来受到全球气候变化的影响准噶尔盆地地区气候格局发生改变,盆地 相比于往年降水和气温明显升高,这种改变影响了盆地的植被变化同时也会在部分地区诱发土壤盐渍化灾害。土壤盐渍化是 我国西北地区常见的导致植被退化的因素,其生成原因与地形和气候因素有关。为了探究准噶尔盆地植被变化与土壤盐渍化 的关联性,基于 2002-2019 年生长季 MOD09A1 遥感影像数据,利用最大值合成法、Mann-Kendall 趋势分析, Hurst 趋势分析法、 相关性分析等方法对准噶尔盆地植被和土壤盐渍化变化趋势以及两者的关联性进行了分析。结果表明,受区域降水和气温升 高的影响,近 20 年来准噶尔盆地生长季植被整体呈增加趋势, 各季节增加区域面积占比为 $63.50 \%$ - $90.93 \%$, 平均为 $77.98 \%$ 。 土壤盐渍化呈减少趋势, 各季减少区域面积占比为 $46.50 \%-86.78 \%$, 平均为 $70.68 \%$ 。在地形低洼、排水不畅的区域土壤盐渍 化程度加重, 植被因盐分胁迫导致衰退,植被减少及巨大的蒸发降水比使得该地区土壤进一步变干,湿度降低。关联性分析结 果表明各季植被与土壤盐渍化的变化中呈显著负相关的区域面积占比为 $37.36 \%$ - $57.83 \%$,平均为 $51.75 \%$ 。 Hurst 趋势预测结 果表明,当前植被和土壤盐渍化两者呈显著性变化的区域未来预测与当前变化方向相同,两者呈一般性变化的区域未来预测与 当前相反。研究有助于在全球气候变化背景下了解准噶尔盆地近年来生态环境变化,结果为区域生态环境的可持续发展提供 参考。
\end{abstract}

关键词:植被;土壤盐渍化;变化趋势;相关性分析

\section{Analysis of negative correlation between vegetation and soil salinization in Junggar Basin}

DU Bingchenxi, CHENG Yongxiang* , WU Ling

College of Life Science, Shihezi University, Shihezi 832000, China

\begin{abstract}
As the main climate unit in Northern Xinjiang, the environmental change of Junggar basin will affect the overall ecological environment change of Northern Xinjiang. Vegetation, as an important indicator of regional ecological environment, which directly reflects the ecological situation of Junggar basin. In recent years, under the influence of global climate change, the climate pattern of Junggar basin has changed. Compared with previous years, the precipitation and temperature in the basin have increased significantly. This change affects the vegetation change in the basin, and also induces soil salinization in some areas. Soil salinization is a common factor leading to vegetation degradation in Northwest China, which is related to terrain and climate factors. In order to explore the relationship between vegetation change and soil salinization in Junggar basin, this study was based on MOD09A1 remote sensing image data during the growing season from 2002 to 2019, this study analyzed the variation trend of plant cover and soil salinization in Junggar Basin and the correlation between them by using of the methods of maximum value composite, Mann-Kendall trend analysis, Hurst trend analysis and
\end{abstract}

基金项目: 欧盟 Erasmus+项目 (598838-EPP-1-2018-EL-EPPKA2-CBHE-JP)

收稿日期: 2020-09-24; 网络出版日期:2021-07- 24

*通讯作者 Corresponding author.E-mail: chengyongxiang_613@163.com 
correlation analysis. The results showed that in the last 20 years, the plant cover in Junggar Basin was increasing in the growing season due to the influence of the regional precipitation and climate warming. The proportion of the increased area in each season was $63.50 \%-90.93 \%$, with an average of $77.98 \%$. Soil salinization, however, showed a decreasing trend, with a decreased area of $46.50 \%-86.78 \%$ in each season and the average value was $70.68 \%$. In the low-lying area with poor drainage, the degree of soil salinization increased, the vegetation declined due to salt stress, the decrease of vegetation and the huge ratio of evaporation and precipitation made the soil further dry and the humidity decreased. The correlation analysis indicated that the proportion of areas with significantly negative correlation between vegetation and soil salinization in each season were $37.36 \%-57.83 \%$, with an average of $51.75 \%$. And the results of Hurst trend prediction suggested that the future prediction direction of areas with significant change in aspects above are the same as the current change trend, while one with a general change is opposite to the current change direction in future direction. This study devoted to obtain the information of the ecological environment changes in Junggar Basin in recent years under the global climate change background, in order to provide references for the sustainable development of regional ecological environment.

Key Words : vegetation; soil salinization; trend change; correlation analysis

植被是联结土壤、大气和水分等要素的自然纽带, 对气候变化敏感 ${ }^{[1]}$ 。近年来受全球气候变化的影响, 中国西北地区正由暖干型向暖湿型气候转变 ${ }^{[2]}$ 。这种气候变化对地处干旱半干旱区的准噶尔盆地植被影响 十分显著。近 60 年来,盆地所在区域年均气温和降水量分别以 $0.35{ }^{\circ} \mathrm{C} /(10 \mathrm{a})$ 和 $13.25 \mathrm{~mm} /(10 \mathrm{a})$ 的速率增 加 ${ }^{[3]}$, 并且降水多集中在一年中的两端 ${ }^{[4]}$ 。气候特点及其当前变化趋势理论上有利于盆地短命植物和旱生 植物的生长和繁殖。相关研究表明自 2000 年以来盆地内古尔班通古特沙漠短命植物覆盖度随着年降水量的 增加而增加 ${ }^{[5]}$, 旱生植物也呈现缓慢上升趋势 ${ }^{[6]}$ 。但鸣军等 ${ }^{[7]}$ 在分析近年来准噶尔盆地植被整体变化时 发现, 虽然盆地植被总体呈增加趋势, 但仍有近 33.0\%的区域植被呈现退化趋势, 目前学者对这些区域植被退 化的成因尚无明确解释。

另有研究表明,准噶尔盆地中的一些低平地和矮沙丘因为近年来表土盐分升高,致使其上梭梭幼苗萌发 受到抑制, 种群发生衰退 ${ }^{[8-9]}$ 。王飞等 ${ }^{[10]}$ 以新疆塔里木盆地南缘为研究区, 利用 NDVI、SI 二者之间的关系构 建特征空间, 表明随着含盐量的增加, NDVI 逐渐降低。陈实等 ${ }^{[11]}$ 基于 MODIS 数据土壤盐渍化遥感监测指数 (SRSI) 对北疆 2000-2014 土壤盐渍化状况进行监测分析,结果表明北疆农区土壤盐渍化整体呈现降低趋 势。何宝忠 ${ }^{[12]}$ 基于 MOD13A1-NDVI 数据, 探讨土壤盐渍化与植被和地貌类型的关系, 结果表明在植被繁茂 区域多为非盐渍化和轻度盐渍化区,植被稀疏多为重盐渍化区,且高程较低区域的盐渍化现象普遍较重。

综上所述, 土壤盐渍化变化是否是驱动当前准噶尔盆地植被整体趋好,局部退化的间接原因,目前尚缺乏 详细研究。对盆地低洼聚盐区是否会随着当前气候变化出现 “盐增植减” 的假设也尚未进行过证明。因此, 研究拟通过 SRSI 及 NDVI 等生态因子趋势分析来反映盆地植被及土壤盐渍化动态变化, 探索盆地近年来气 候-盐渍化-植被变化间的因果关系。实现对准噶尔盆地植被及土壤盐渍化变化趋势及二者相关性的准确描 述, 结果为荒漠植被保护提供一定的科学依据。

\section{1 研究区概况}

准噶尔盆地位于中国新疆北部, 地理坐标为 $42^{\circ} 36^{\prime} 18^{\prime \prime}-48^{\circ} 39^{\prime} 30^{\prime \prime} \mathrm{N}, 82^{\circ} 17^{\prime} 44^{\prime \prime}-96^{\circ} 1^{\prime} 13^{\prime \prime} \mathrm{E}$, 东北为阿尔 泰山, 南为天山山脉, 西侧为准噶尔西部山地, 盆地呈三角形, 是中国第二大的内陆盆地, 面积约为 38 万 $\mathrm{km}^{2}$, 海拔 189-1569 m, 东高西低,如图 1 所示。盆地中部有我国面积最大的固定、半固定沙漠-古尔班通古特沙 漠。按照区域 13 个气象站点 1951-2019 年观测数据统计准噶尔盆地年均温为 $2-11{ }^{\circ} \mathrm{C}$, 年降水量为 $20-$ $295 \mathrm{~mm}$, 日降水量 $\geqslant 0.1 \mathrm{~mm}$ 累积日数年平均值为 $14-107 \mathrm{~d}$, 相对湿度年平均值为 $33 \%-62 \%$, 年日照时数平 均值为 2542-3335 $\mathrm{h}$ 。准噶尔盆地植被呈非紧缩型分布, 代表植物有梭梭 (Haloxylon ammodendron (C. A. 
Mey.) Bunge)、白梭梭 (Haloxylon Persicum Bunge ex Boiss. Et Buhse)、琵琶柴(Reaumuria songonica ( PalL) Maxim.)、柽柳( Tamarix chinensis Lour.)、盐穗木 (Halostachys caspica (Bieb.) C. A. Mey.) 和早春短命植物等。 盆地土壤以棕钙土和荒漠灰钙土为主, 局部为龟裂土、草甸土和盐碱土等土壤类型。

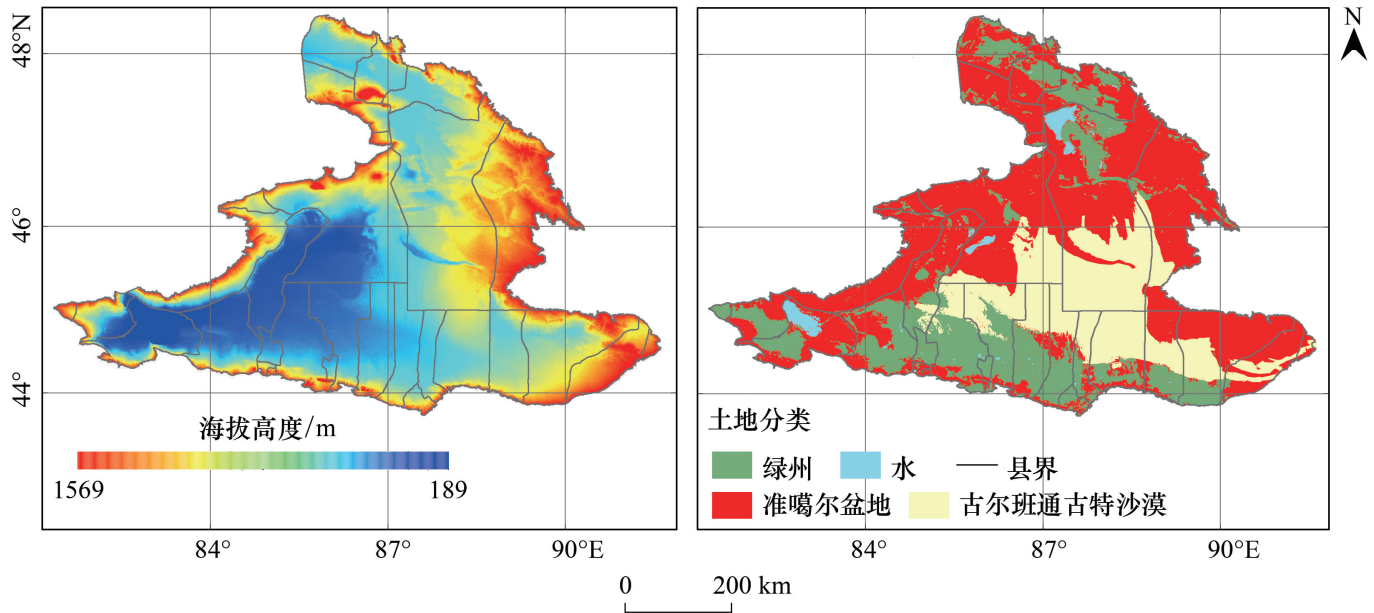

图 1 研究区介绍

Fig.1 Introduction to the research area

\section{2 研究数据与方法}

\section{1 研究数据及预处理}

研究所用数据包括中国农业统计数据平台 ( http://hx. acmr. com.cn/) 获取的相应县、市耕地播种面积数 据。中国气象科学数据共享服务网 (http://data.cma.cn/) 下载获得的 1951-2019 年准噶尔盆地十三个气象 站月值数据,该数据用于研究区气候特征的描述。美国国家航空航天局官网( https://ladsweb.modaps.eosdis. nasa.gov) 下载获得的 2002-2019 年 MOD09A1 数据 (MODIS Terra 500m 地表反射率 8 天合成数据产品), 数 据轨道号为 h23v04、h24v04, 共获取 1824 幅遥感影像, 将下载后的影像利用 MRT( MODIS Reprojection Tools) 软件进行图像拼接和投影转换 (椭球体 WGS- 84,投影 Mercator)。通过 MOD09A1 地表反射率 $500 \mathrm{~m}$ 状态信 息, 获取每幅影像晴空像元归一化植被指数( NDVI) 、土壤盐渍化指数( SRSI) 和土壤湿度指数( SIMI)。

$$
\begin{aligned}
\text { NDVI }= & \left(\rho_{\text {nir }}-\rho_{\text {red }}\right) /\left(\rho_{\text {nir }}+\rho_{\text {red }}\right) \\
& S I=\sqrt{\rho_{\text {blue }} \times \rho_{\text {red }}} \\
\text { SRSI }= & \sqrt{(\mathrm{NDVI}-1)^{2}+S I^{2}} \\
\text { SIMI }= & 0.7071{\sqrt{\rho_{\text {swir } 1}{ }^{2}+\rho_{\text {swir } 2}{ }^{2}}}^{2}
\end{aligned}
$$

式中 $\rho_{\text {nir }} \rho_{\text {red }} \rho_{\text {blue }} \rho_{\text {swirl }}$ 和 $\rho_{\text {swir } 2}$ 分别代表 MOD09A1 中的近红外、红、蓝、短波红外 1 和短波红外 2 波段。

基于指标计算,研究按照季节变化结合 MODIS 数据日期来确定统计时间范围春季( 3 月 6 日-5 月 25 日)、夏季 $(5$ 月 26 日 -8 月 29 日)、秋季( 8 月 30 日一 11 月 25 日)、生长季( 3 月 6 日- 11 月 25 日,运用最大 值合成法 (Maximum Value Composite, MVC) 得到每年春、夏、秋和生长季 NDVI 的最大值。在 SRSI 最大值统 计时间范围选取中, 研究为避免准噶尔盆地春、秋积雪对结果的影响, 最大值统计时间范围选择春季(4 月 15 日-5 月 25 日)、夏季( 5 月 26 日-8 月 29 日)、秋季( 8 月 30 日-10月 15 日)、生长季( 4 月 15 日一 10 月 15 日) 的遥感影像参与相应 SRSI 最大值合成。为排除雪山与河流、湖泊等高 SRSI 变异区域对结果的影响, 研究 利用 SRSI 变异系数小于 $90 \%$ 的条件对上述地类进行了去除,剩余研究区被用于结果分析。

另外, 研究下载了 2002-2019 年 MODIS Terra $6 \mathrm{~km}$ 月值地表温度 (MOD11B3) 和 $500 \mathrm{~m}$ 年值土地分类数 
据(MCD12Q1), 热带测雨观测计划 (Tropical Rainfall Measuring Mission, TRMM) $0.25^{\circ}$ 月值降水等辅助分析数 据。以上数据经图像预处理,数据投影信息与 MODIS09A1 相同。因 MOD11B3 和 TRMM 数据主要用于结果 区域正确性检验,故研究未改变其各自数据的空间分辨率。

\section{2 研究方法}

\subsubsection{Mann-Kendall 趋势分析}

研究对盆地各季节 NDVI 和 SRSI 时间序列进行 Mann-Kendall 趋势变化分析,其检验公式为 ${ }^{[13]}$ :

$$
\begin{gathered}
S=\sum_{i=2}^{n} \sum_{j=1}^{i-1} \operatorname{sign}\left(X_{i}-X_{j}\right) \\
\operatorname{sign}()=\left\{\begin{array}{c}
-1 X_{i}-X_{j}<0 \\
0 X_{i}-X_{j}=0 \\
1 X_{i}-X_{j}>0
\end{array}\right.
\end{gathered}
$$

$S$ 为正态分布, 其均值为 0 , 方差 $\operatorname{Var}(S)=n(n-1)(2 n+5) / 18$ 。当统计量 $S$ 取值不同时,算公式分 别为:

$$
\left\{\begin{array}{cc}
Z=(S-1) / \sqrt{n(n-1)(2 n+5) / 18} & S>0 \\
Z=0 & S=0 \\
Z=(S+1) / \sqrt{n(n-1)(2 n+5) / 18} & S<0
\end{array}\right.
$$

在双边趋势检验中, 对于给定的置信水平 $\alpha$, 若 $|Z| \geqslant Z_{1-\alpha / 2}$, 即在置信水平 $\alpha$ 上, 时间序列数据存在明 显的上升或下降趋势。 $Z$ 为正值表示增加趋势, 零值表示不变, 负值表示减少趋势。本研究中当 $\alpha$ 为 $95 \%$ 置 信水平时, $Z$ 的取值为 1.96 。指标变化趋势的等级划分如表 1 所示。

表 1 指标变化趋势等级划分表

Table 1 Grade division table of index change trend

\begin{tabular}{lcccc}
\hline $\begin{array}{l}\text { 统计值 } \\
\text { Statistics }\end{array}$ & $\begin{array}{c}\text { 变化等级 } \\
\text { Change level }\end{array}$ & $\begin{array}{c}\text { 缩写 } \\
\text { Abbreviation }\end{array}$ & $\begin{array}{c}\text { 备注 } \\
\text { Remarks }\end{array}$ \\
\hline$Z \geqslant 1.96$ & 增加 & 显著增加 & SII & 2 \\
$0<Z<1.96$ & & 一般增加 & SLI & 1 \\
$Z=0$ & & 不变 & CON & 0 \\
$-1.96<Z<0$ & 减少】 & 一般减少 & SLD & -1 \\
$Z \leqslant-1.96$ & & 显著减少 & SID & -2 \\
\hline
\end{tabular}

\subsubsection{Hurst 指数趋势预测}

为进一步探讨 NDVI 和 SRSI 时间序列未来可能的变化趋势, 研究利用 Hurst 趋势分析预测了两组序列未 来的变化方向。Hurst 指数计算过程为 ${ }^{[14]}$ :

对于时间序列 $\left\{\mathrm{Var}_{t}\right\}, t=1,2, \cdots, n$, 首先定义均值序列:

$$
\overline{\operatorname{Var}_{(\tau)}}=\frac{1}{\tau} \sum_{t=1}^{\tau} \operatorname{Var}_{(\tau)} \tau \text { 为大于 } 1 \text { 的正整数 }
$$

其次计算出累计离差, 公式为:

$$
X_{(t, \tau)}=\sum_{t=1}^{t}\left(\operatorname{Var}_{(t)}-\overline{\operatorname{Var}_{(\tau)}}\right)
$$

然后计算出极差,公式为:

$$
R_{(\tau)}=\underbrace{\max }_{1 \leqslant i \leqslant \tau} X_{(t, \tau)}-\underbrace{\min }_{1 \leqslant i \leqslant \tau} X_{(t, \tau)}
$$

再利用如下公式计算标准差： 


$$
S_{(\tau)}=\left[\frac{1}{\tau} \sum_{t=1}^{\tau}\left(\operatorname{Var}_{(t)}-\operatorname{Var}_{(\tau)}\right)^{2}\right]^{\frac{1}{2}} \tau=1,2, \cdots, n
$$

$H$ 为 Hurst 指数,采用最小二乘法和公式 $\log (R / S)_{n}=a+H \times \log n$ 得到 $H$ 值, 该值有以下三种形式: 如果 $0.5<H<1$ 表明变量时间序列是一个持续性序列,即未来变化趋势与过去的变化趋势保持一致,且持续性随 着 $H$ 增大将愈强。如果 $H=0.5$ 则说明变量时间序列为随机序列, 无法根据现有规律预测未来变化, 即不存在 任何长期相关性。如果 $0<H<0.5$, 则表明变量时间序列为反持续性序列, 即未来的变化趋势与过去的变化 趋势相反, $H$ 值越接近 0 , 反持续性越强。

\subsection{3 相关性分析}

研究通过相关系数分析, 探讨 NDVI 和 SRSI 时间序列在各季节的相关关系, 利用相关系数显著性检验 (0.05 水平) 判定二者间关系是否显著,所得结果再结合 NDVI 和 SRSI 趋势变化方向进行综合解释。相关性 计算公式如下:

$$
r_{x y}=\frac{\sum_{i=1}^{n}\left[\left(x_{i}-\bar{x}\right)\left(y_{i}-\bar{y}\right)\right]}{\sqrt{\sum_{i=1}^{n}\left(x_{i}-\bar{x}\right)^{2} \sum_{i=1}^{n}\left(y_{i}-\bar{y}\right)^{2}}}
$$

式中, $r_{x y}$ 表示变量 $x 、 y$ 的相关系数; $n$ 为监测时段的累计年数; $x_{i}$ 表示第 $i$ 年 NDVI 值; $y_{i}$ 表示第 $i$ 年 SRSI 值; $\bar{x} 、 \bar{y}$ 为变量平均值。

\section{3 结果与分析}

\section{1 准噶尔盆地植被及土壤盐渍化季节变化规律分析}

研究将 2002-2019 年 NDVI 和 SRSI 春、夏、秋三季平均值的结果进行排序,探讨植被和表土盐渍化的季 节消长, 由图 2 可知, 盆地绿洲农作物年生长峰值出现在夏季 (夏绿型), 古尔班通古特沙漠植被年生长峰值 出现在春季 (春绿型), 地势相对低洼 (多盐生植物生长区) 其上植被年生长峰值出现在秋季(秋绿型), 研究 结果与各类群植被生长规律相吻合 ${ }^{[15]}$ 。盆地土壤盐渍化情况则表现为春季峰值型最为普遍, 夏、秋次之。对 比图 2 中两幅图, 可得同一空间中植被生长和土壤盐渍化的峰值发生季节在时间上不重叠, 表明二者峰值呈 负关联,即表土盐分高的季节不利于植被的生长。

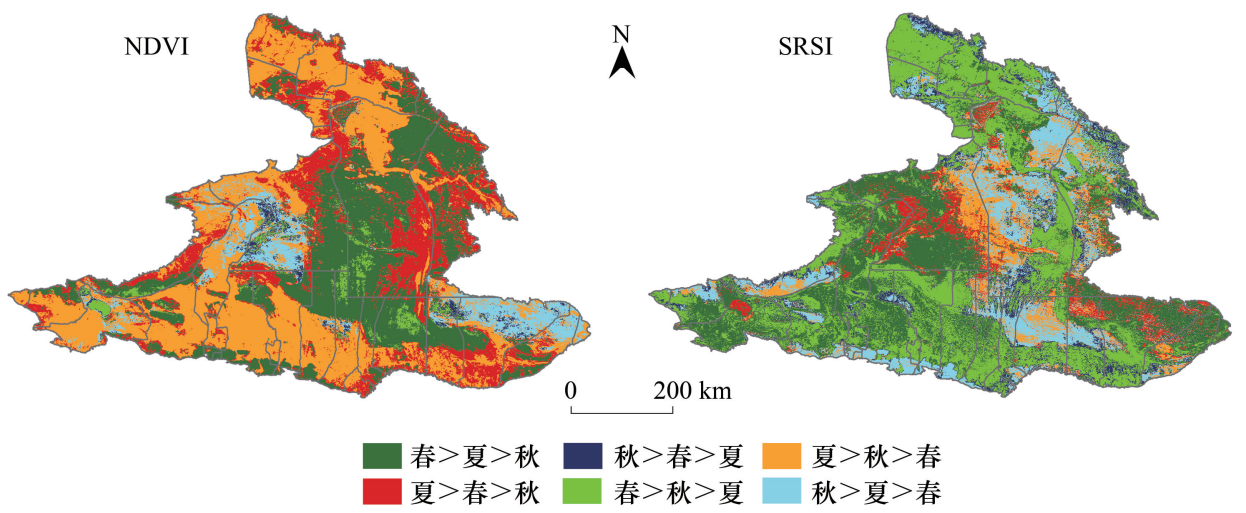

图 2 准噶尔盆地春、夏、秋三季 NDVI 和 SRSI 平均值排序结果图

Fig.2 Ranking results of NDVI and SRSI average values in spring, summer and autumn in Junggar Basin NDVI: 归一化植被指数, SRSI: 土壤盐渍化指数

3.2 准噶尔盆地植被与土壤盐渍化的趋势变化分析

研究对 2002-2019 年盆地不同季节 NDVI 和 SRSI 时间序列分别进行 Mann-Kendall 趋势分析, 并按表 1 
进行等级划分, 结果如图 3 所示, 盆地 NDVI 整体呈增加趋势, 局部呈减趋势, 各季节 NDVI 呈增加趋势的面积 占比在 63.50\%一 -90.93\% 之间, 平均为 $77.98 \%$ (图 4)。而盆地 SRSI 整体呈减少趋势, 局部呈增加趋势, 各季 节 SRSI 呈减少趋势的面积占比在 46.50\%- $86.78 \%$ 之间,平均为 $70.68 \%$ (图 5)。春季是盆地植被增加及土 壤盐渍化减少面积占比都最大的一个季节。

盆地中古尔班通古特沙漠各季节 NDVI 呈增加趋势的面积占比在 65.00\%-88.20\% 之间, 平均为 $76.79 \%$, 其中春季 NDVI 呈增加趋势的面积占比最高(图 4)。分析原因与沙漠区域近年来春季降水增加, 沙地表面聚
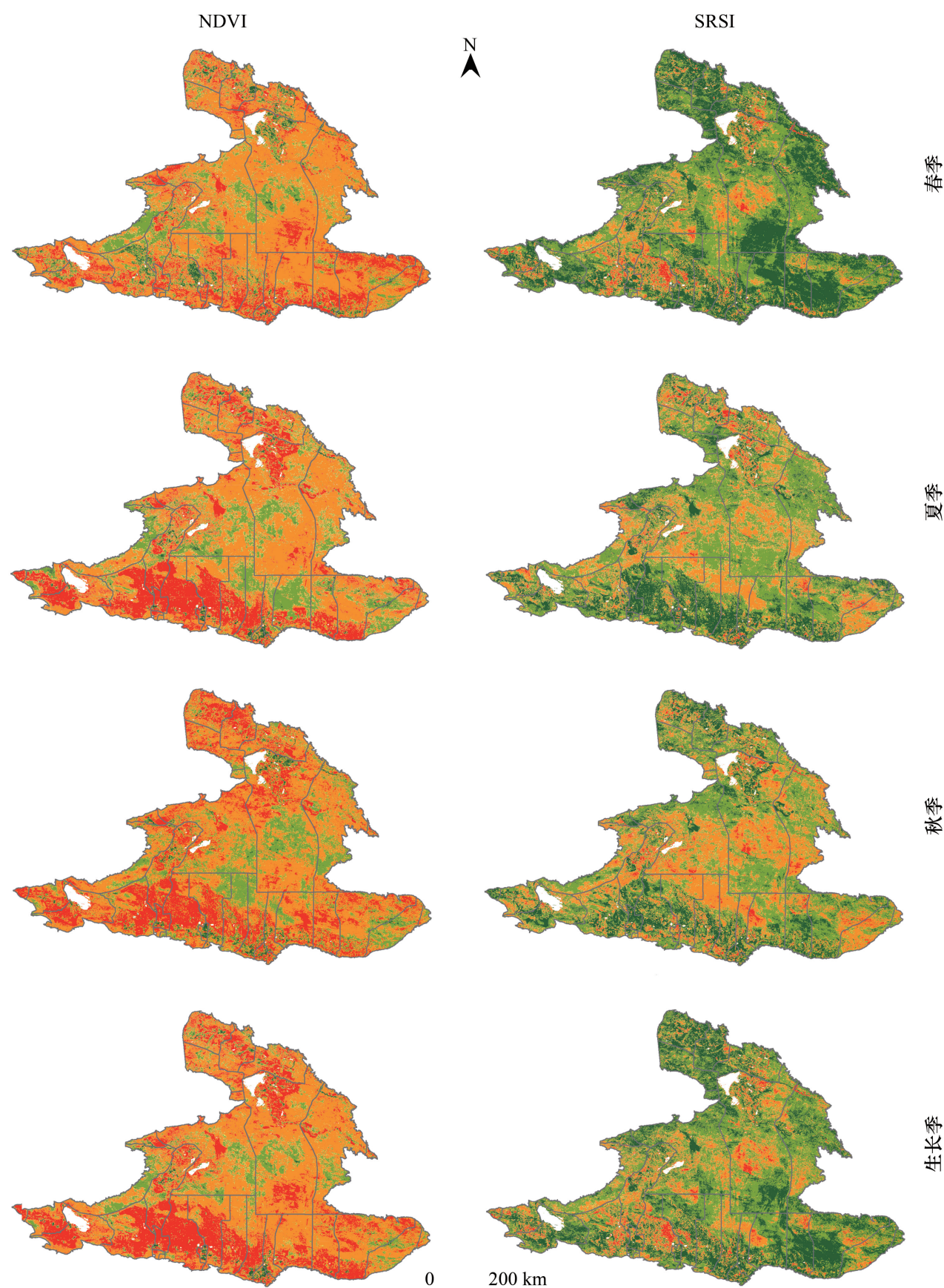

显著增加 $\quad$ 一般增加 不变
显著减少

图 3 准噶尔盆地各季节 NDVI 和 SRSI Mann-Kendall 变化趋势等级划分结果图

Fig.3 Ranking results of seasonal NDVI and SRSI Mann-Kendall trend in Junggar Basin 
盐能力差, 短命植物大量生长繁殖密切相关 ${ }^{[16]}$ 。盆地中绿洲区域各季节 NDVI 呈增加趋势的面积占绿洲总 面积的 $63.50 \%$ - $90.93 \%$,平均为 $81.56 \%$ 。滴灌技术的广泛运用使得绿洲区域大面积盐碱地、荒漠、草地转变 为耕地, 极大的增加了盆地内耕地面积, 绿洲变的越来越绿 ${ }^{[17]}$ 。盆地荒漠区各季节 NDVI 呈增加趋势的面积 占比为 $71.54 \%-78.48 \%$, 平均为 $76.30 \%$ 。

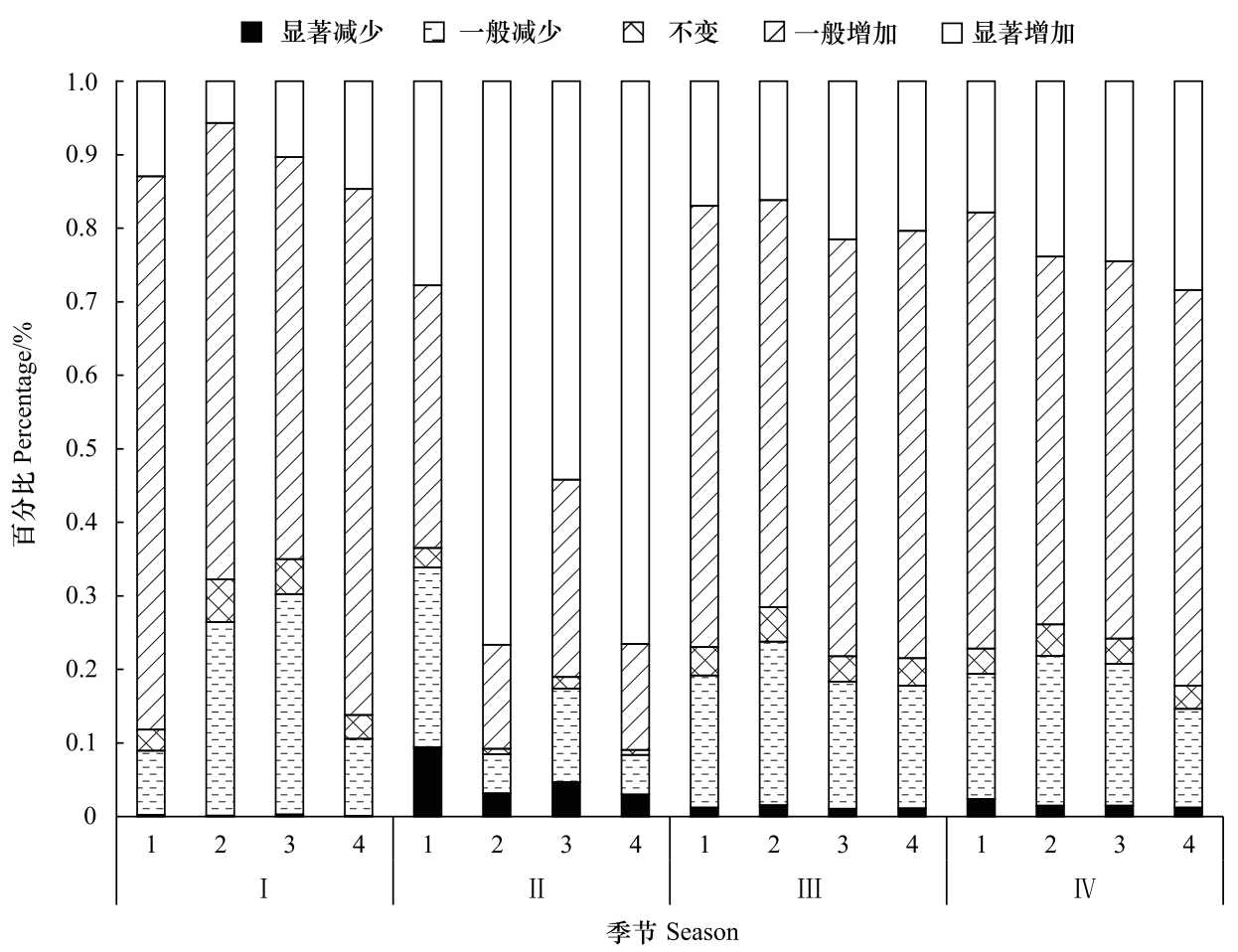

图 4 2002-2019 年准噶尔盆地 NDVI 各等级变化趋势面积占比分析

Fig.4 Analysis on the proportion of NDVI change trend area of each rank in Junggar basin from 2002 to 2019

1: 春季 Spring；2: 夏季 Summer； 3: 秋季 Fall； 4: 生长季 Growing Seasons. I : 古尔班通古特沙漠 Gurbantunggut desert；II : 绿洲 Oasis; III : 荒漠区 Desert area; IV : 准噶尔盆地 Junggar basin. SID: 显著减少 Significant decrease; SLD : 一般减少 Slight decrease; CON : 不变 Constant; SLI : 一般增加 Slight increase; SII : 显著增加 Significant increase

盆地春季 SRSI 呈增加趋势的面积占比最小, 为总面积的 $16.13 \%$, 其次为夏季 $26.72 \%$, 最后为秋季 36.33\% (图 5)。分析秋季盆地 SRSI 呈增加趋势的面积占比高的原因, 与近年来秋季气温升高、降水增加密切 相关 ${ }^{[18]}$ 。沙漠和荒漠区 SRSI 呈增加趋势的面积占比排序与盆地整体规律一致,而绿洲区该面积占比排序则 为春季 $<$ 秋季<夏季,这与绿洲夏季农作物生长峰值规律相吻合。

研究对盆地各季节 NDVI 和 SRSI 时间序列分别进行 Hurst 趋势预测分析,结果如图 6 所示, 从图中可以 看出, 植被和土壤盐渍化指数的未来变化趋势总体表现为当前两者呈显著增加的区域未来仍会继续增加, 当 前两者呈显著减少的区域未来仍会继续减少,但一些当前变化趋势不显著的区域,Hurst 指数未来变化趋势预 测结果与当前变化趋势方向相反。

\section{3 植被与土壤盐渍化的相关性分析}

研究将 SRSI 和 NDVI 发生趋势变化且相关性分析为显著的区域求交集, 得到二者存在趋势变化且关系 密切的区域,结果如图 7 所示, 准噶尔盆地大部分区域近 20 年均表现为 “盐减植增” 模式,其次盆地范围地势 相对较低的区域表现为 “盐增植减” 模式, 各研究时段两种模式的面积合计占盆地总面积的 37.36\%一 $57.83 \%$, 平均为 $51.75 \%$ (图 8), 结果表明土壤盐渍化程度与植被生长呈负相关关系。盆地中仅有较少区域表 现为“盐减植减”或“盐增植增”模式。 


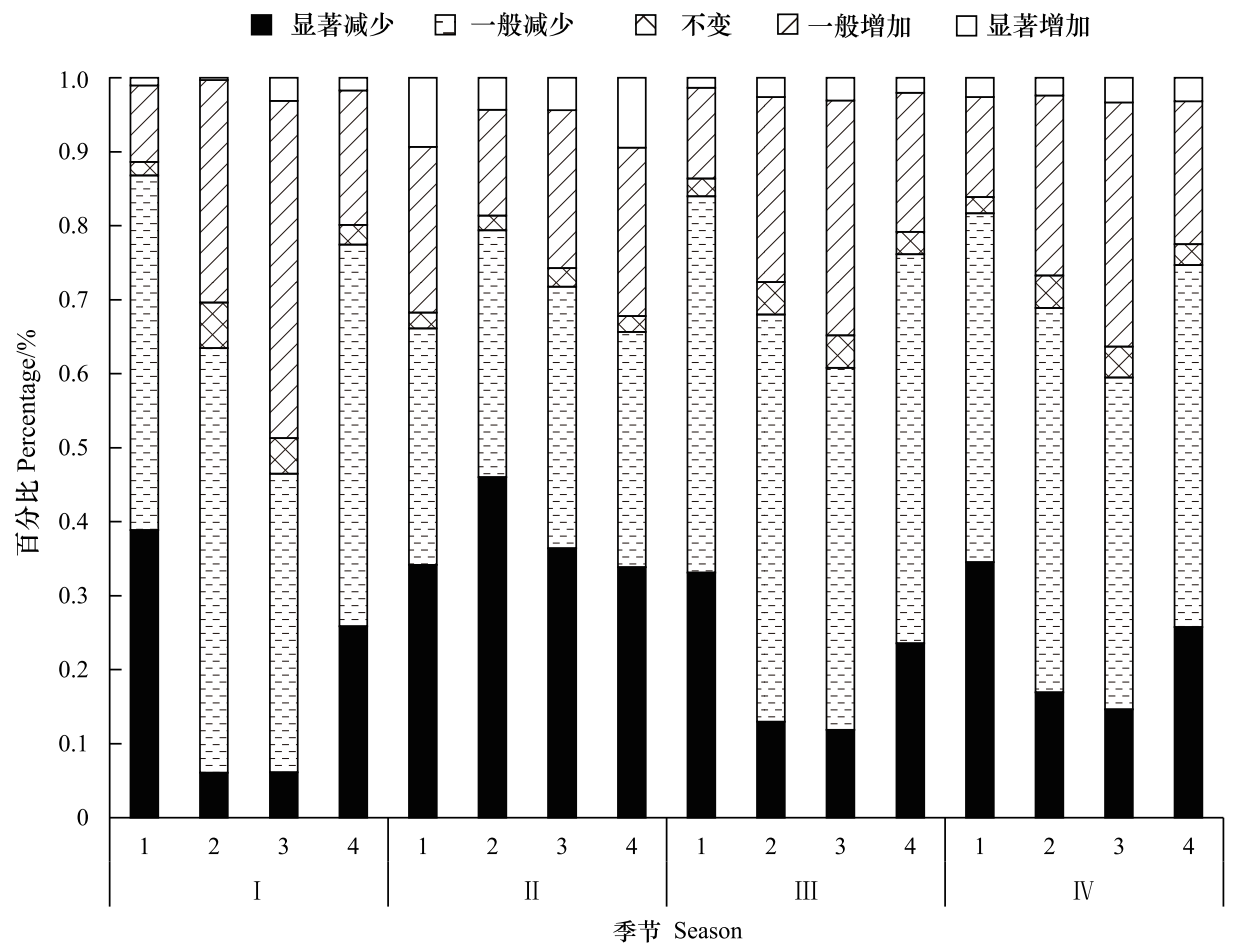

图 $52002-2019$ 年准噶尔盆地 SRSI 各等级变化趋势面积占比分析

Fig.5 Analysis on the area proportion of SRSI change trend of different rank in Junggar basin from 2002 to 2019

1: 春季 Spring; 2: 夏季 Summer; 3: 秋季 Fall; 4: 生长季 Growing seasons. I : 古尔班通古特沙漠 Gurbantunggut desert；II : 绿洲 Oasis; III : 荒漠区 Desert area; IV : 准噶尔盆地 Junggar basin. SID: 显著减少 Significant decrease; SLD : 一般减少 Slight decrease; CON : 不变 Constant; SLI : 一般增加 Slight increase; SII : 显著增加 Significant increase

表 2 准噶尔盆地各季节 SRSI 和 NDVI 二者存在趋势变化且呈显著相关的区域面积统计 $/\left(10^{4} \cdot \mathrm{km}^{2}\right)$

Table 2 SRSI and NDVI in different seasons of Junggar basin, which have a trend change and a significant correlation in regional area statistics

\begin{tabular}{|c|c|c|c|c|}
\hline $\begin{array}{l}\text { 项目 } \\
\text { Items }\end{array}$ & $\begin{array}{l}\text { 春季 } \\
\text { Spring }\end{array}$ & $\begin{array}{c}\text { 夏季 } \\
\text { Summer }\end{array}$ & $\begin{array}{l}\text { 秋季 } \\
\text { Fall }\end{array}$ & $\begin{array}{c}\text { 生长季 } \\
\text { Growing seasons }\end{array}$ \\
\hline SRSI $\searrow N D V I \searrow$ & 1.52 & 2.12 & 0.86 & 0.91 \\
\hline $\mathrm{SRSI} \nearrow \mathrm{NDVI} \searrow$ & 2.54 & 4.03 & 5.1 & 1.48 \\
\hline $\mathrm{SRSI} \searrow \mathrm{NDVI} \nearrow$ & 18.1 & 16.67 & 16.28 & 12.33 \\
\hline $\mathrm{SRSI} \nearrow \mathrm{NDVI} \nearrow$ & 1.08 & 2.58 & 4.37 & 2.39 \\
\hline 总面积 Total area & 23.25 & 25.41 & 26.61 & 17.11 \\
\hline
\end{tabular}

フ: 增加 Increase; 】: 降低 Decrease.; NDVI:归一化差异植被指数 Normalized Difference Vegetation Index; SRSI: 土壤盐渍化遥感监测指数 Salinization Remote Sensing Index

沙漠区除秋季外,春、夏及生长季“盐减植增”的区域面积占比均超过沙漠显著相关区域面积 $56.76 \%$,其 中春季 “盐减植增”模式面积占比最高,达到沙漠显著相关区域面积的 $85.51 \%$ 。绿洲区域夏季“盐减植增” 区 域面积占比最高,达到整个绿洲区显著相关区域面积的 $89.51 \%$,而绿洲生长季 “盐增植增” 变化模式占比最 高为 $35.01 \%$ (图 8), 这充分体现了人类活动对农业生产环境的调控作用。荒漠区各季节 SRSI 与 NDVI 呈负 相关的区域面积占比与盆地整体相当。

\section{4 讨论}

相关分析表明由于厄尔尼诺一南方涛动引发的暖湿气流变化和中纬度西风带对水汽的输送,最终导致北 

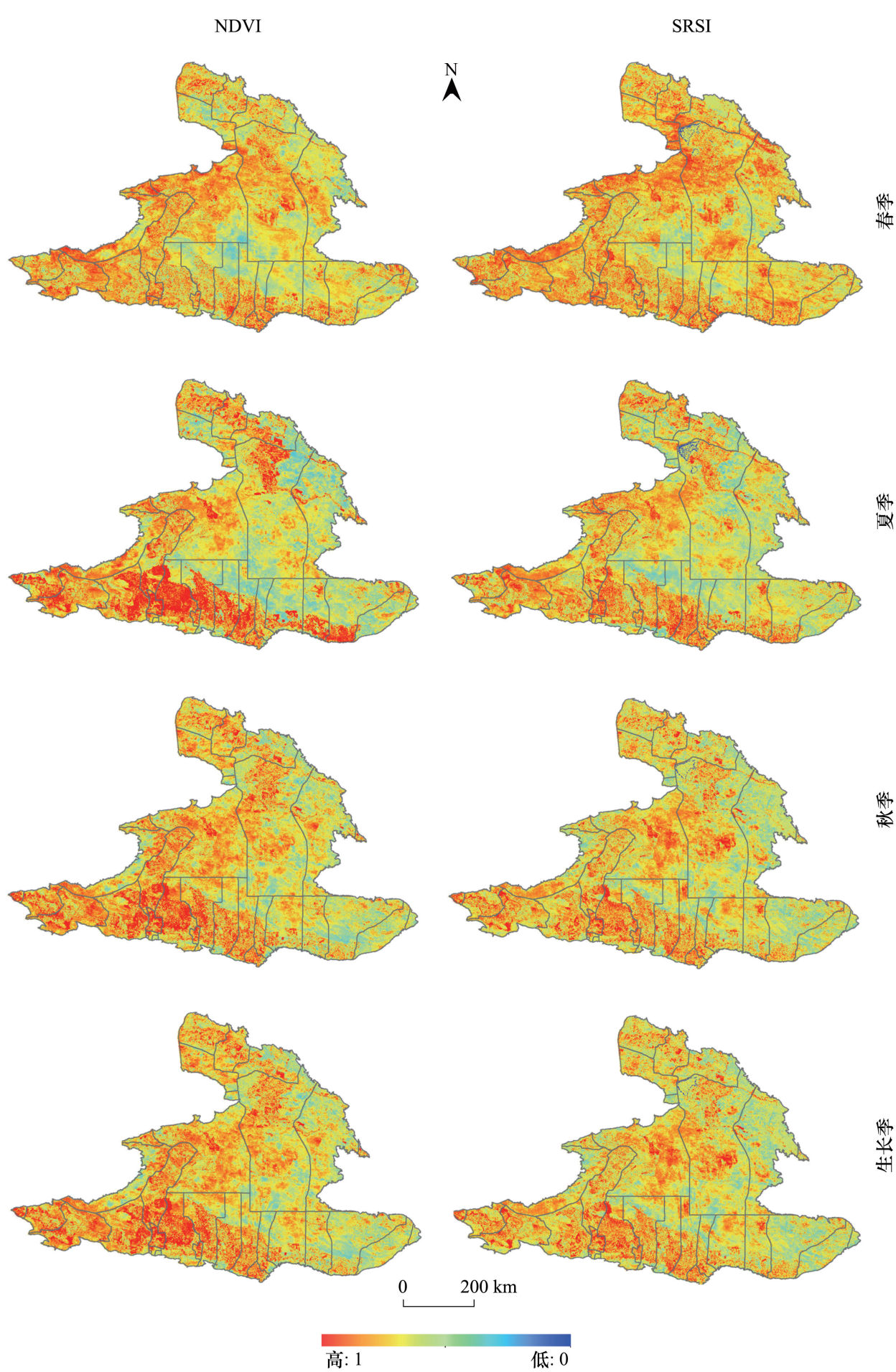

图 6 2002-2019 年准噶尔盆地各季节 NDVI 和 SRSI Hurst 变化趋势预测图

Fig.6 Prediction of NDVI and SRSI Hurst future change trend in different seasons of Junggar basin from 2002 to 2019

疆降水量增加 ${ }^{[19-22]}$ 。为了解不同季节降水对植被的影响,研究利用 TRMM 降水数据分析了 2002-2019 年盆 地各季节降水格局的变化。从图 9 可知盆地春、秋两季降水增加较为明显,夏季降水增加相对较少,该结果与 Zhang ${ }^{[23]}$ 等所得北疆地区降水量在冷季比暖季增加更快的结论一致。降水量及冷季降水的增加总体上有利 于植被的生长和繁殖。降水格局变化在本研究表现为盆地各季节有 $63.50 \%-90.93 \%$ 的植被呈增加变化趋 

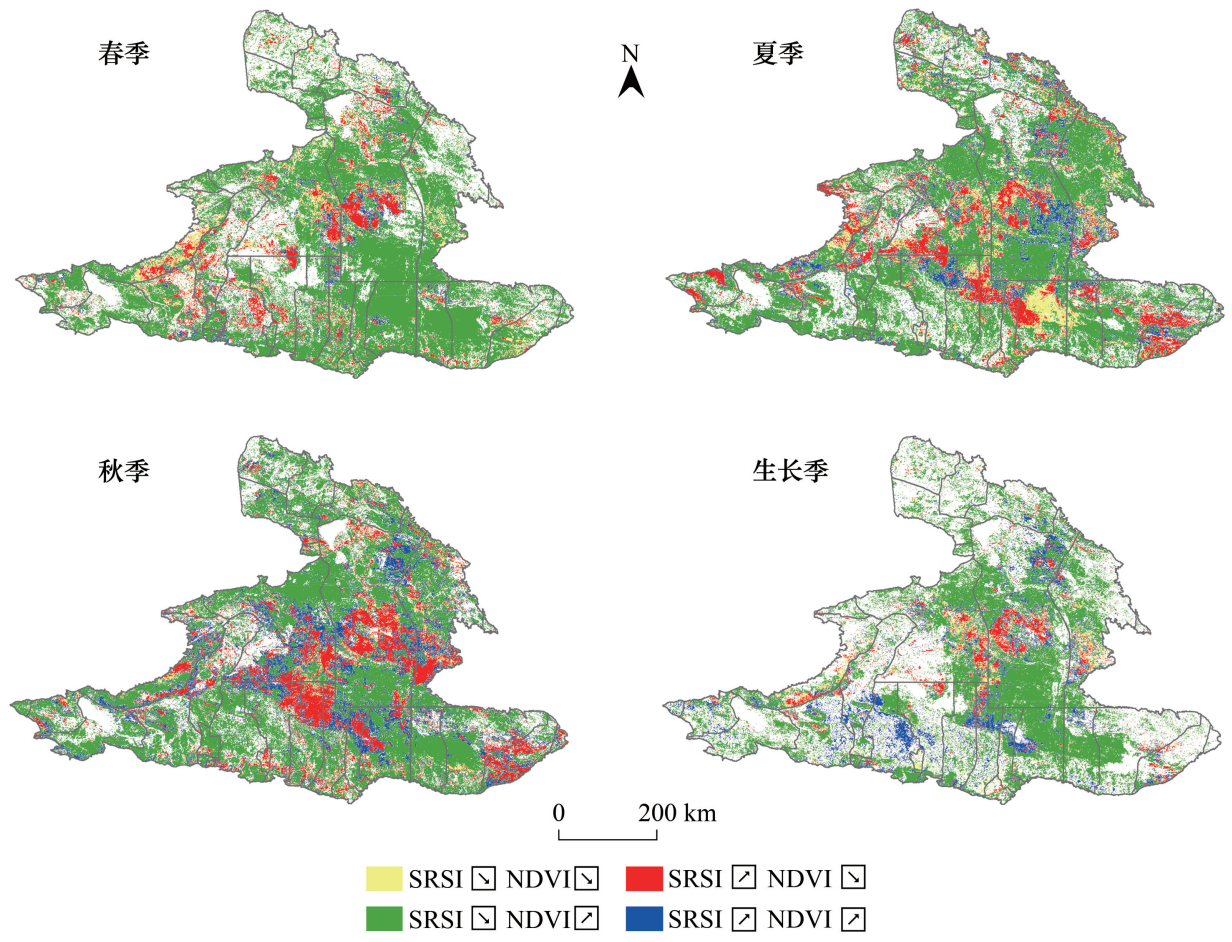

图 7 准噶尔盆地各季节 SRSI 和 NDVI 二者存在趋势变化且呈显著相关的区域分布图

Fig.7 The result distribution diagram of SRSI and NDVI in different seasons of Junggar basin, which have a trend change and a significant correlation

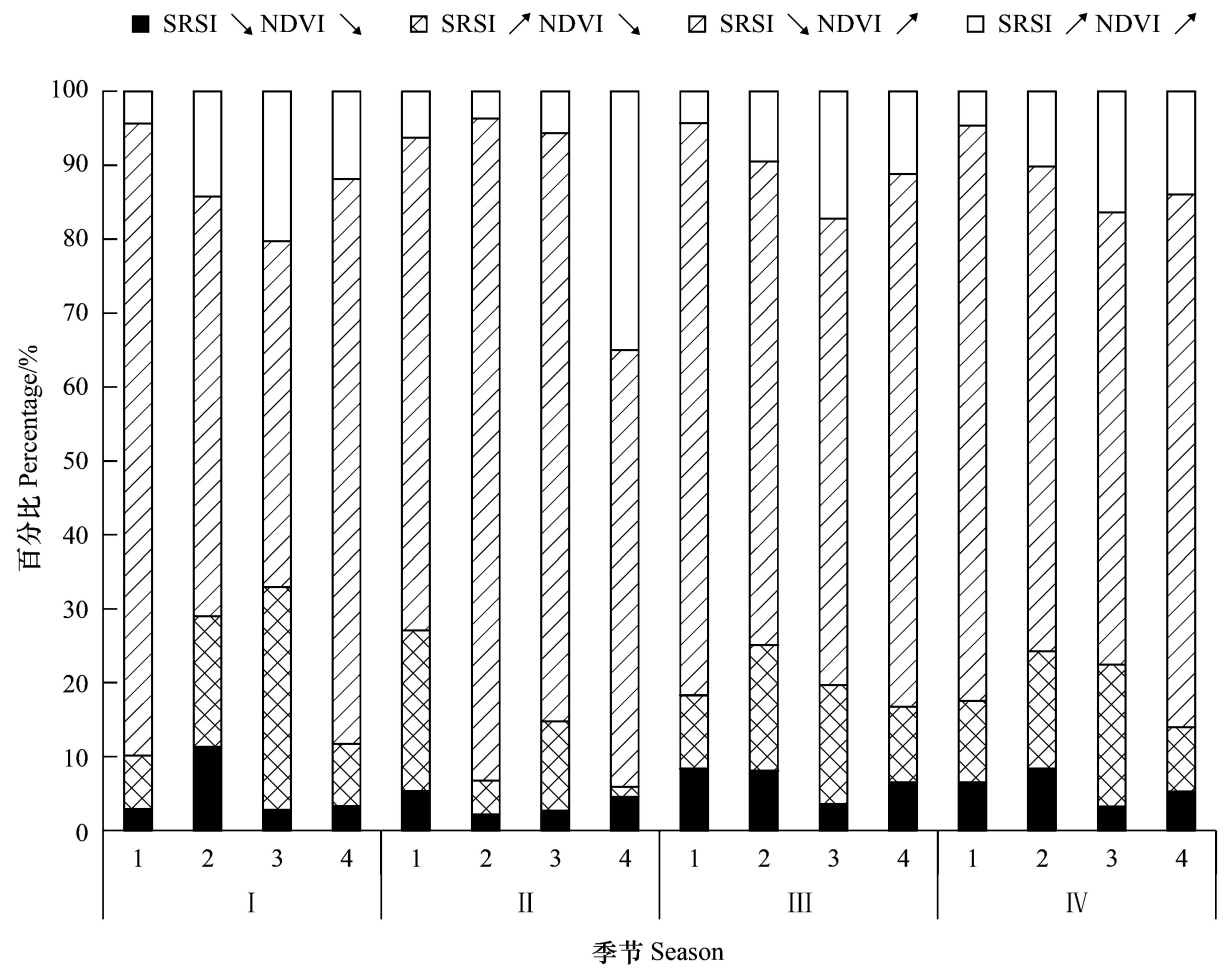

图 8 准噶尔盆地 SRSI 和 NDVI 发生趋势变化且相关性分析为显著的区域各类型面积百分比

Fig.8 Area percentage of each type that SRSI and NDVI have tendency changes and significant correlation in Junggar Basin $\nearrow$ : 增加 Increase; 】: 降低 Decrease. 1: 春季 Spring; 2: 夏季 Summer; 3: 秋季 Fall; 4: 生长季 Growing seasons. I : 古尔班通古特沙漠 Gurbantunggut desert； II : 绿洲 Oasis; III : 荒漠区 Desert area; IV : 准噶尔盆地 Junggar basin 
势。该结果与近年来盆地旱生植物呈现缓慢上升变化趋势 ${ }^{[6]}$, 沙漠短命植物覆盖度变化及萌发机制等研究 结论相吻合 ${ }^{[24]}$ 。
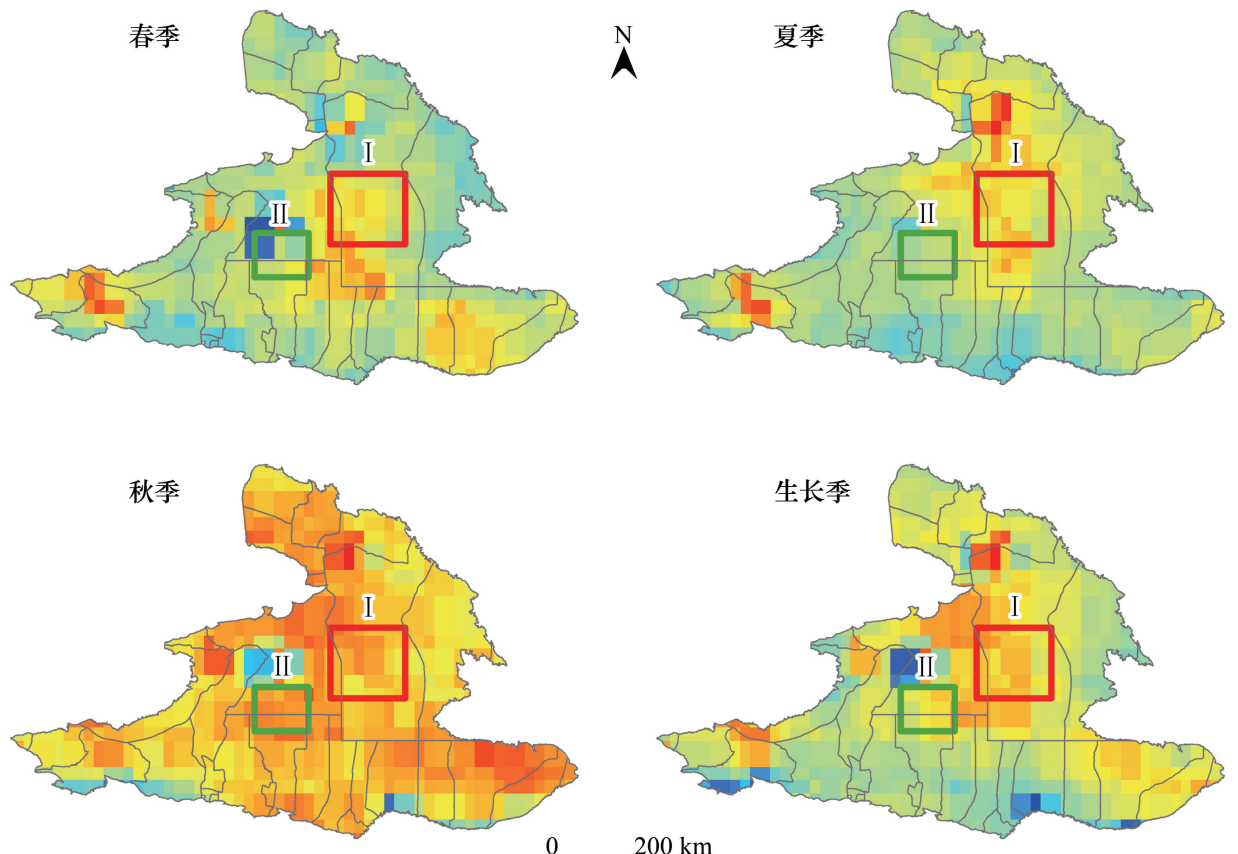

高: 4.00

低: -4.00

图 $92002-2019$ 年准噶尔盆地季节性降水的 Mann-Kendall 结果分析图

Fig.9 Mann-Kendall analysis of seasonal precipitation in Junggar basin from 2002 to 2019

区域温度的升高和降水的增加导致一些地势相对较低的地区 (低平地、低洼处及河水汇流处) 土壤盐分 增加。Taghizadeh-Mehrjardi 等 ${ }^{[25]}$ 研究表明高程、坡度等地形参数会对土壤盐渍化的聚集有重大的影响。卢 磊 ${ }^{[26]}$ 等认为地势高低变化会影响土壤盐渍化的分布格局,一般地势较低处的土壤盐分相对高。对比研究结 果可知,近年来盆地表土盐分增加区均为地势相对低洼的区域 (图 1、图 3)。这些区域常年排水不畅, 受近年 来降水增加的影响,地表温度会下降 ${ }^{[27-28]}$, 气温升高, 区域巨大的蒸发降水比 ${ }^{[29]}$ 使得这些低洼处在降水后表 土易于再次变干, 土壤毛管水作用增强,盐分表聚,盐胁迫导致植被生长不良,植被退化,植被的退化导致土壤 表面无法保存水分使得该地区的土壤湿度逐渐下降。为证明该推断,完善结果证据链,研究利用遥感土壤湿 度指数和地表温度趋势分析, 选取土壤盐渍化对植被影响最为典型的两个区域给予论证(图 10), 样区 I 和 II 分别位于福海县的中部和和布克赛尔蒙古自治县与玛纳斯县交界处。两个样区的地形均易发生土壤盐渍 化, 受气候变化影响, 上述地区 2002-2019 年降水升高 (图 9), 地表温度降低, 配合地形和土壤因素, 这些地 区均表现为土壤盐渍化增加, 植被因盐分胁迫无法正常生长发育导致植被覆盖下降, 同时, 土壤湿度与植被覆 盖呈正相关, 在空间分布上具有较好的一致性 ${ }^{[30-31]}$, 植被覆盖的降低使得该地区土壤无法保持湿润状态土壤 逐渐变干(图 10)。以上分析通过闭合证据链的方式证明了盆地中的积水聚盐区植被因盐而退化的结果正 确, 回答了近年来盆地植被虽总体增加,但仍有部分区域呈现退化的主要成因。

人为因素是导致盆地绿洲植被变化的主要因素。研究基于 MCD12Q1 全球植被分类方案 1 和统计数据, 分析 2002-2019 年盆地绿洲变化,结果与研究所得该区植被呈显著增加的结论相一致(图 11)。虽然盆地绿 洲一些特殊积水地形也表现为春季土壤盐分呈较明显增加趋势, 但受区域农业生产模式转变的影响,特别是 滴灌使得灌头下土壤水分接近饱和, 水分扩散后形成一个半圆锥形的土壤浸润体, 土壤盐分随水移动后, 盐被 淋洗到浸润体的外围, 结果土壤耕作层盐分下降 ${ }^{[32]}$, 促进了农作物生长。这是绿洲盐碱地在生长季呈现 “盐 

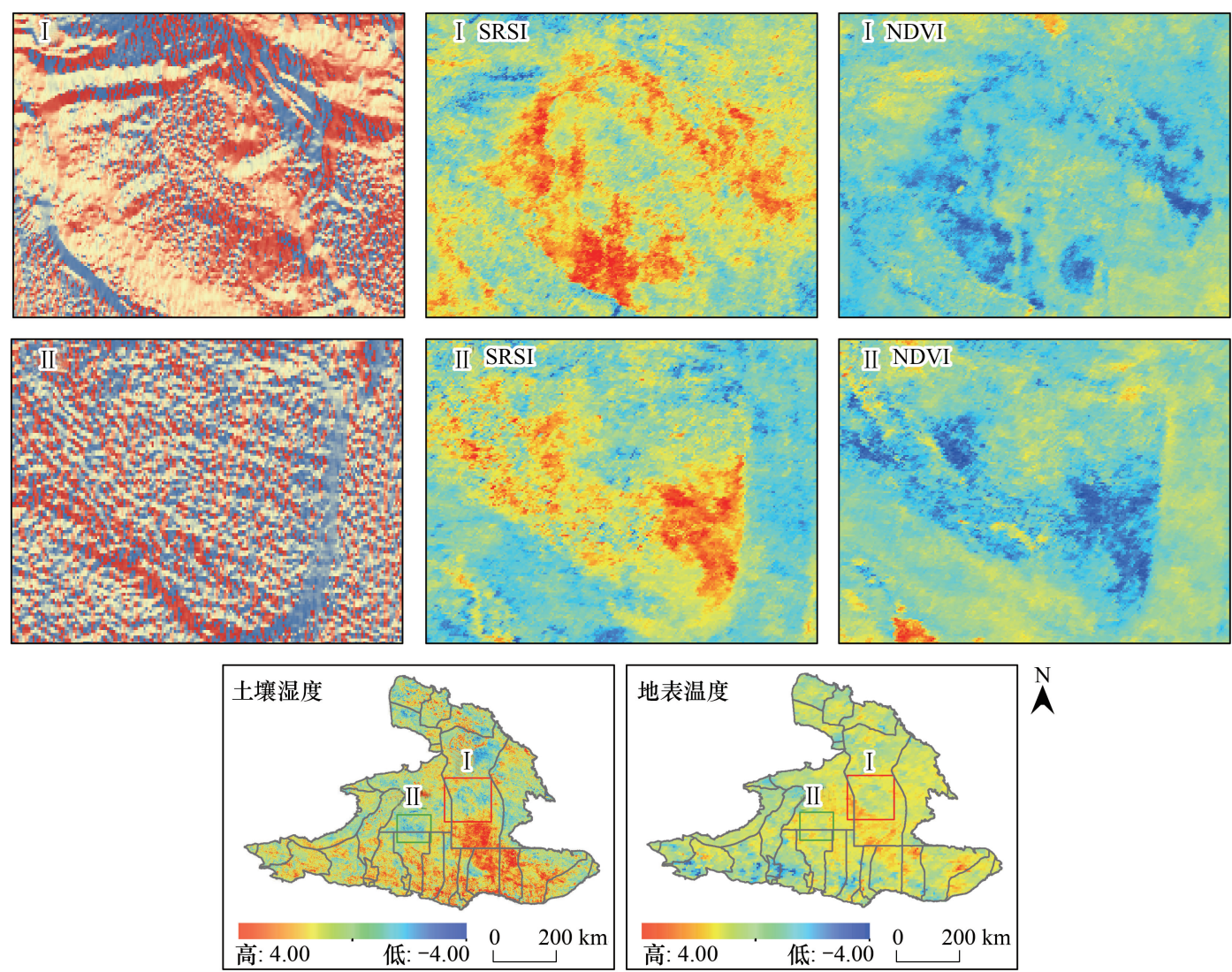

图 10 准噶尔盆地“盐增植减”结果验证分析图

Fig.10 Validation analysis chart of “SRSI increase and NDVI decrease” results in Junggar Basin
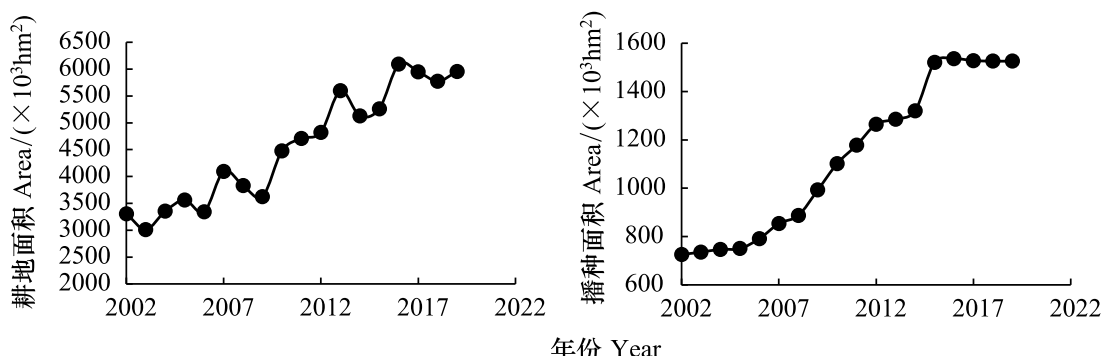

图 $112002-2019$ 年准噶尔盆地耕地及播种面积变化

Fig.11 Changes of cultivated land and sown area in Junggar basin from 2002 to 2019

增植增”的内在原因。

\section{5 结论}

受区域降水和气温升高的影响,近 20 年来准噶尔盆地各季节植被整体呈增加趋势, 增加区域面积占比为 $63.50 \%$ - $90.93 \%$, 平均为 $77.98 \%$ 。土壤盐渍化呈减少趋势, 减少区域占比为 $46.50 \%$ - $86.78 \%$, 平均为 $70.68 \%$ 。但在地形低洼、排水不畅的区域土壤盐渍化程度加重, 植被减少。关联性分析结果表明植被与盐渍化 变化趋势普遍呈负相关, 其中二者存在趋势变化且又呈显著负相关的区域面积占盆地总面积的 $37.36 \%-$ $57.83 \%$,平均为 $51.75 \%$ 。气候变化驱动的土壤盐渍化变化成为影响当前准噶尔盆地植被变化的重要生态因子。 


\section{参考文献 (References) :}

[ 1 ] Thuiller W, Lavorel S, Araújo M B, Sykes M T, Prentice I C. Climate change threats to plant diversity in Europe. Proceedings of the National Academy of Sciences of the United States of America, 2005,102(23) : 8245-8250.

[2] 商沙沙, 廉丽姝, 马婷, 张琨, 韩拓. 近 54-a 中国西北地区气温和降水的时空变化特征. 干旱区研究, 2018, 35(1): 68-76.

[ 3 ] He B B, Sheng Y, Cao W, Wu J H. Characteristics of climate change in Northern Xinjiang in 1961-2017, China. Chinese Geographical Science, $2020,30(2): 249-265$.

[ 4] 阿帕尔. 肉孜, 叶尔克江 - 霍依哈孜, 高培, 高彦兵, 刘海红. 新疆近 $50 \mathrm{a}$ 气候的变化特征. 南京信息工程大学学报: 自然科学版, 2014, $6(4): 356-362$.

[ 5 ] Jia Y Y, Sun Y, Zhang T, Shi Z Y, Maimaitiaili B, Tian C Y, Feng G. Elevated precipitation alters the community structure of spring ephemerals by changing dominant species density in Central Asia. Ecology and Evolution, 2020, 10(4) : 2196-2212.

[ 6 ] 蒋超亮, 吴玲, 安静, 刘丹, 王绍明. 古尔班通古特沙漠旱生植物时空分布特征. 生态学报, 2019, 39(3): 936-944.

［７］ 万鸣军, 夏朝宗. 1982-2013 年准噶尔盆地植被长势变化分析. 林业资源管理, 2016, (5): 39-46.

[ 8 ] 刘斌, 刘形, 李否, 赵新俊, 韩志全, 马大伟. 古尔班通古特沙漠西部梭梭大面积退化原因. 生态学杂志, 2010, 29(4) : 637-642.

[ 9 ] 赵从举, 康慕谊, 雷加强. 准噶尔盆地典型地段植物群落及其与环境因子的关系. 生态学报, 2011, 31(10): 2669-2677.

［10］王飞，丁建丽，伍漫春. 基于 NDVI-SI 特征空间的土壤盐渍化遥感模型. 农业工程学报，2010，26(8)：168-173.

[11] 陈实, 徐斌, 金云翔, 黄银兰, 张文博, 郭剑, 申格, 杨秀春. 北疆农区土壤盐渍化遥感监测及其时空特征分析. 地理科学, 2015,35 (12) : 1607-1615.

[12］何宝忠, 丁建丽, 王飞, 张喆, 刘博华. 基于物候特征的盐渍化信息数据挖掘研究. 生态学报, 2017, 37(9): 3133-3148.

[13] He L Y, Meng X Y, Du J, Wang Y J, Cai Y G, Tang X L. Study on the ecological characteristics and change analysis of Xin Jiang Junggar Basin Based on the NDVI. Nature Environment and Pollution Technology, 2014, 13(1): 191-196.

[14] Zhao X, Tan K, Zhao S. Fang J. Changing climate affects vegetation growth in the arid region of the northwestern China. Journal of Arid Environments, 2011, 75(10): 946-952.

［15］张衷华, 宋晓倩, 唐中华, 梁正伟. 中国盐生植物系统发育多样性及省域差异性. 植物研究, 2020, 40(3) : 330-338.

[16] 段呈, 吴玲, 王绍明, 贺凌云. 近 30 年古尔班通古特沙漠短命植物的时空格局. 生态学报, 2017, 37(8): 2642-2652.

[17］王丹, 吴世新, 张寿雨. 新疆 20 世纪 80 年代末以来耕地与建设用地扩张分析. 干旱区地理, 2017, 40(1) : 188-196.

[18] 张扬, 楚新正, 杨少敏, 郭超. 近 56-a 新疆北部地区气候变化特征. 干旱区研究, 2019, 36(1) : 212-219.

[19] Huang W, Chang S Q, Xie C L, Zhang Z P. Moisture sources of extreme summer precipitation events in North Xinjiang and their relationship with atmospheric circulation. Advances in Climate Change Research, 2017, 8(1): 12-17.

[20] Lu B, Li H Y, Wu J, Zhang T X, Liu J, Liu B, Chen Y, Baishan Jiazila. Impact of ElNiño and Southern Oscillation on the summer precipitation over Northwest China. Atmospheric Science Letters, 2019, 20(8) : e928.

[21] Wang W G, Li H Y, Wang J, Hao X H. Water vapor from western Eurasia Promotes Precipitation during the Snow Season in Northern Xinjiang, a typical arid region in Central Asia. Water, 2020, 12(1):141.

[22] Zhou Y, Huang A N, Zhao Y, Yang Q, Jiang J, La M K. Influence of the sea surface temperature anomaly over the Indian Ocean in March on the summer rainfall in Xinjiang. Theoretical and Applied Climatology, 2015, 4 119(3/4) : 781-789.

[23] Zhang Y H, Xie P, Pu X, Xia F Q, An J L, Wang P, Mei Q W. Spatial and temporal variability of drought and precipitation using cluster analysis in Xinjiang, Northwest China. Asia-Pacific Journal of Atmospheric Sciences, 2019, 55(2) : 155-164.

[24] Duan C, Wu L, He L Y, Wang S M. Spatio-temporal distribution pattern of vegetation coverage in Junggar Basin, Xinjiang. Acta Ecologica Sinica, $2016,36(2): 72-76$.

[25] Taghizadeh-Mehrjardi R, Minasny B, Sarmadian, Malone B P. Digital mapping of soil salinity in Ardakan region, central Iran. Geoderma, 2014, $213: 15-28$

[26] 卢否, 乔木, 周生斌, 间俊杰. 新疆渭干河流域土壤盐渍化及其驱动力分析. 农业现代化研究, 2011, 32(3): 357-361.

[27] 张述文, 刘源, 曹帮军, 李少英. GLDAS 和 CMIP5 产品的中国土壤湿度一降水耦合分析及变化趋势. 气候与环境研究, 2016, 21(2)： 188- 196 .

[28］李艳, 侯金亮, 黄春林. 基于 Copula 函数的地表温度空间降尺度研究. 遥感技术与应用, 2017, 32(5): 818-824.

[29] 张静. 准噶尔盆地表生生态环境演化及驱动力分析 $[\mathrm{D}]$. 西安: 长安大学, 2016.

[30］王生春. 基于多源数据的三江源土壤湿度时空变化及生态效应研究 [D] 青海:.青海大学, 2020.

[31］翟雅倩，张棚，周旗，常宵移. 秦巴山区植被覆盖与土壤湿度时空变化特征及其相互关系. 地球信息科学学报, 2018, 20(7)：967-977.

[32] 王振华, 杨培岭, 郑旭荣, 何新林, 张金珠, 李文吴. 新疆现行灌溉制度下膜下滴灌棉田土壤盐分分布变化. 农业机械学报, 2014, 45 (8) : 149-159. 